Reprod. Nutr. Dévelop., 1984, 24 (1), 11-19.

\title{
Variations annuelles du comportement d'œstrus, du taux et des possibilités d'ovulations chez la brebis Peulh du Niger
}

\author{
A. YENIKOYE
}

Ecole Supérieure d'Agronomie, Université de Niamey, B.P. 10960 Niamey, Niger.

Summary. Annual variations in estrous behavior, ovulation rate and the possibility to ovulate in Niger ewes of the Peulh breed.

Annual variations in estrous and ovulation behavoir were studied in 23 bicolored Peulh ewes for a period of 30 months. The percentages of estrus and ovulation shown over a year of observation were 76 and $86 \%$, respectively. The average rate of ovulation was $1.3 \pm 0.04(\bar{X} \pm$ SEM)

Reproductive activity was minimal from January to April, as the percentages of estrus and ovulation varied between 43.8 and $61 \%$ and between 53.1 and $86 \%$, respectively. This period was marked by the advent of anestrus, interrupted frequently by silent and irregular ovulations. Anestrus lasted an average of $81.9 \pm 9.8$ days $(\bar{X} \pm$ SEM).

Reproductive activity was maximal from May to December, as the percentages of estrus and ovulation varied between 77 and $97 \%$ and between 87.5 and $100 \%$, respectively. During this period ovarian activity was interrupted by prolonged diestrus wich lasted an average of $24.9 \pm 3.3$ days $(\bar{X} \pm$ SEM).

The ovulation rate did not differ significantly $(P>0.05)$ between periods of minimal and maximal sexual activity.

These results suggest that the reproductive potentialities of Peulh sheep on a good diet are comparable to those of certain ovine breeds in temperate zones. The results also suggest that anestrus in bicolored Peulh sheep is probably a different physiological process than the one observed in some breeds of sheep in which anestrus is marked by total ovarian inactivity.

\section{Introduction.}

L'ancestrus saisonnier et le taux d'ovulations sont deux facteurs connus dont dépendent les potentialités de reproduction chez la brebis (Gunn et Doney, 1979). La variation saisonnière de l'activité sexuelle est une caractéristique commune à plusieurs races ovines (Hafez, 1952 ; Wheeler et Land, 1977 ; Mauléon, 1980). Chez la brebis peulh, Yenikoye et al. (1982) ont observé sur un faible effectif d'animaux des corps jaunes persistants, le plus souvent en juin, et des cycles à phase folliculaire prolongée de décembre à avril. Si l'existence de ces deux types 
d'anomalies de la fonction ovarienne est confirmée chez la brebis peulh elle aura pour conséquence une baisse de la fertilité comparable à celle qui est observée chez certaines races de moutons européens.

Les variations saisonnières du taux d'ovulations chez la brebis n'ont été mises en évidence que chez les races à taux d'ovulations élevé (taux supérieur à 2) (Land et al., 1973 ; Wheeler et Land, 1977 ; Valls et al., 1977). Elles se traduisent par une diminution graduelle du nombre d'ovulations lors de la transition entre saison sexuelle et ancestrus. Nous ne disposons actuellement d'aucune information sur le taux d'ovulations de la brebis nigérienne.

Le but de ce travail est d'une part de déterminer les variations saisonnières du taux d'ovulations, si elles existent, chez la brebis peulh bicolore, d'autre part d'examiner l'importance des anomalies de la fonction ovarienne dans un troupeau en précisant leur durée et leur fréquence d'apparition.

\section{Matériel et méthodes.}

Deux expériences ont été réalisées dans un parc de l'Université de Niamey (latitude $13^{\circ} 30^{\prime} \mathrm{N}$ ) sur des brebis de race peulh bicolore.

La ration alimentaire de ces animaux a été maintenue constante pendant toute la durée de l'expérience. Elle se composait de fourrage sec (Echinochloa Stagnina), de son de riz, de graines de coton, de tourteau d'arachide, de sel et d'eau à volonté.

$1^{\circ}$ Expérience /. - L'étude des variations annuelles du comportement d'œstrus et des possibilités d'ovulations a été faite de décembre 1980 à novembre 1981, sur un lot de 23 brebis. L'âge des animaux variait entre 18 mois et 4 ans environ et leur poids entre $28,5 \mathrm{~kg}$ et $58 \mathrm{~kg}$ en début d'expérience. Les évolutions des poids vifs des femelles n'ont pas été enregistrées pendant toute la durée de l'expérience.

La détection de l'œestrus a été réalisée 2 fois par jour, le matin entre $8 \mathrm{~h} 30$ et $10 \mathrm{~h}$ et l'après-midi entre $16 \mathrm{~h}$ et $17 \mathrm{~h}$, à l'aide de 2 béliers utilisés alternativement et munis d'un tablier non marqueur. Le critère du comportement d'œstrus est défini par l'immobilité de la brebis pendant le chevauchement du mâle (Thimonier et Mauléon, 1969).

Les possibilités d'ovulations ont été vérifiées par le dosage quotidien de la progestérone plasmatique dans le sang périphérique.

Les prises de sang sont effectuées tous les jours entre $7 \mathrm{~h} 30$ et $8 \mathrm{~h} \mathrm{30}$, au niveau de la veine jugulaire. Le sang prélevé est immédiatement centrifugé et le plasma recueilli, stocké à - $15{ }^{\circ} \mathrm{C}$ jusqu'à la réalisation du dosage radioimmunologique (Yenikoye et al., 1981).

$2^{\circ}$ Expérience $/ 1$. - Le taux d'ovulations moyen a été déterminé et l'existence d'une variation saisonnière de ce taux, testée chez huit brebis âgées de 3 à 4 ans appartenant au lot de l'expérience I. Cette étude a été réalisée de juin 1979 à juillet 1980 . 
L'ovulation est contrôlée par endoscopie sous anesthésie générale et après jeûne préalable de $24 \mathrm{~h}$ (Thimonier et Mauléon, 1969). Les endoscopies sont effectuées 3-4 jours après l'apparition de l'œestrus. En l'absence d'œstrus, les endoscopies sont réalisées tous les 3 jours après la période présumée de l'œestrus. Le nombre d'ovulations est déterminé par le nombre de corps jaunes observés au moment de l'endoscopie.

$3^{\circ}$ Estimations de l'incidence des anomalies ovariennes sur la reproduction, calcul de l'intervalle entre deux phases lutéales.

a) Connaissant la durée moyenne des cycles cestraux normaux propres à une brebis il est possible d'estimer le nombre théorique d'œstrus qu'elle pourrait avoir pendant la période d'observation s'il n'existait ni prolongation de la phase lutéale, ni allongement de l'intervalle séparant deux phases lutéales consécutives.

II est supposé qu'à tout œstrus est associée une ovulation. Le nombre d'œstrus et d'ovulations réellement observés sont alors exprimés en pourcentage du nombre théoriquement possible.

b) L'intervalle entre deux phases lutéales est calculé de la manière suivante : la concentration moyenne $(\bar{X})$ de progestérone est calculée à partir de toutes les valeurs mesurées le jour de l'œestrus $(N)$ et une bande de confiance du type $\bar{X} \pm 2 \sigma / \sqrt{N}$ contenant $95 \%$ des données ( $\sigma$ écart type des valeurs, $N$ nombre de valeurs), est déterminée. Les valeurs qui n'appartiennent pas à cet intervalle correspondent soit au terme de la sécrétion d'un corps jaune, soit à la sécrétion d'un nouveau corps jaune. La durée de la phase lutéale ainsi que celle de l'intervalle entre deux phases lutéales consécutives sont alors déterminées.

$4^{\circ}$ Tests statistiques. - La signification statistique a été déterminée soit par le test non paramétrique du chi-deux pour comparer les nombres d'ovulations, soit par le test de Cox et Stuart pour la recherche de tendance lors de l'allongement de l'intervalle qui sépare deux phases lutéales consécutives. Le fonctionnement alterné des ovaires droit et gauche a été vérifié par le test de Walf-Wolfowitz (Conover, 1971).

\section{Résultats.}

\section{Expérience /.}

1) Variations annuelles du comportement d'cestrus et du taux d'ovulations. - Les pourcentages moyens d'œestrus et d'ovulations enregistrés par rapport aux nombres théoriques sont respectivement de $76 \%$ et $86 \%$.

II existe donc des arrêts de l'activité cyclique du comportement d'œstrus et de l'ovulation ; ces arrêts sont plus fréquents certains mois de l'année et définissent une période critique (ancestrus) qui s'étend de janvier à avril (fig. 1). Les plus faibles pourcentages d'œestrus et d'ovulations sont observés en mars ; ils sont respectivement de $43,8 \%$ et $53,1 \%$. Les pourcentages d'animaux en œestrus et 
d'animaux ovulant sont respectivement de $47,6 \%$ et $61,9 \%$ à cette période de l'année.

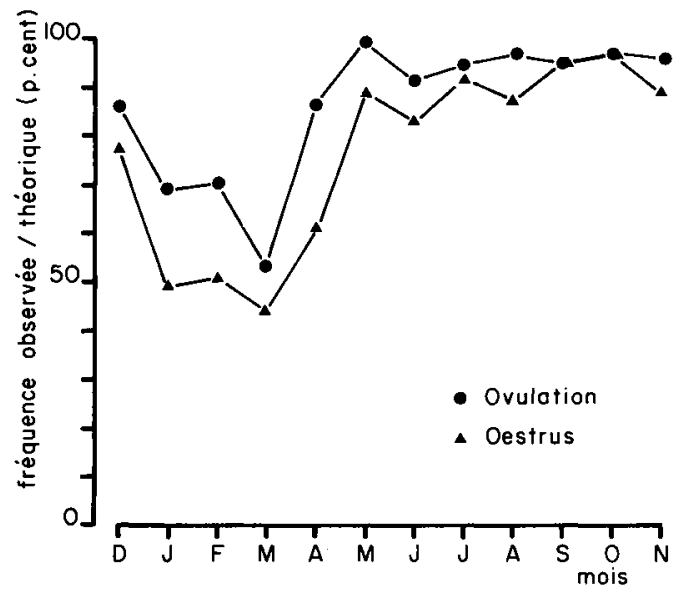

FIG. 1. - Variations saisonnières du comportement d'œestrus et de l'ovulation.

En absence de l'apparition cyclique du comportement d'œstrus et de l'ovulation, on peut noter un allongement soit la phase lutéale, soit de l'intervalle entre 2 phases lutéales consécutives (fig. 2). $76 \%$ des animaux présentent au moins un des deux types d'allongement pendant la période d'observation.

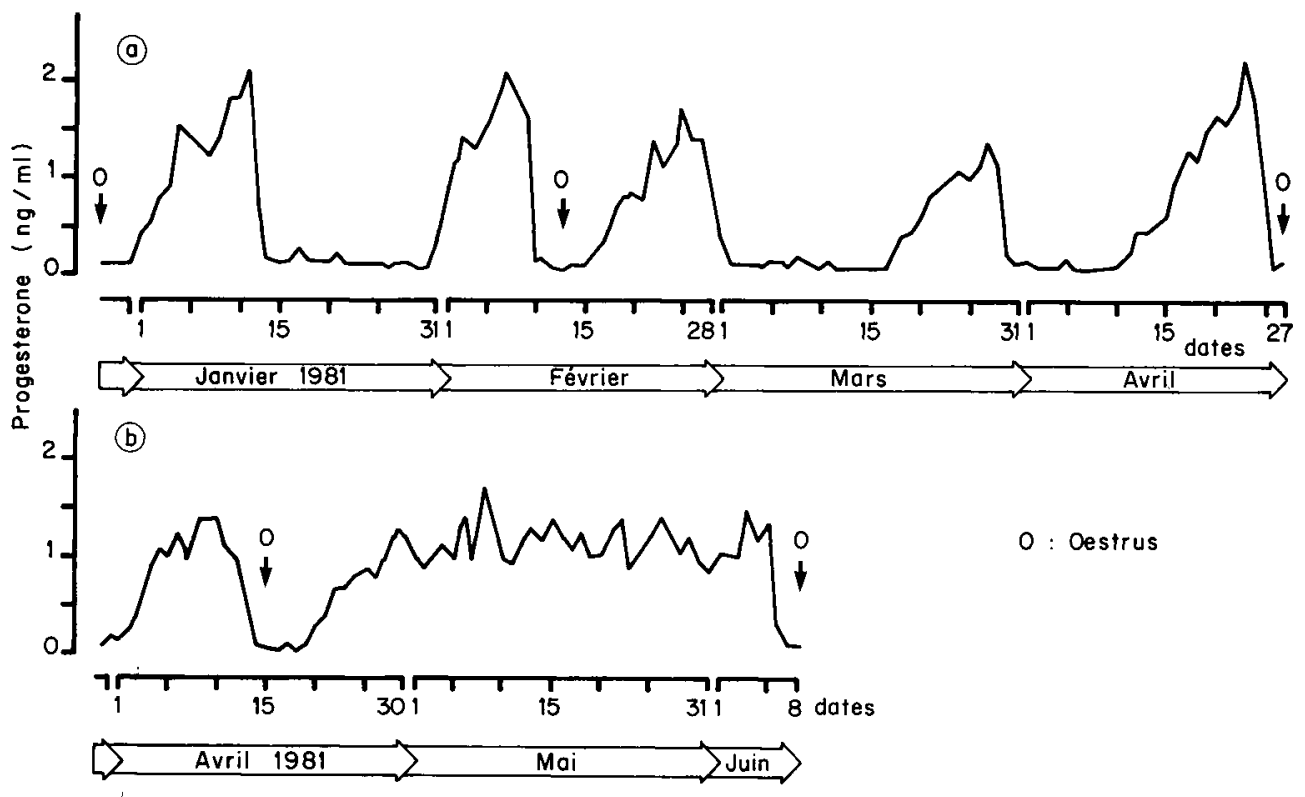

FIG. 2. - Evolution du niveau plasmatique de progestérone au cours : a) de trois allongements de l'intervalle entre deux phases lutéales en janvier, mars et avril ; $\boldsymbol{b}$ ) d'un cycle à phase lutéale prolongée. 
2) Variations et importance de l'allongement de la phase lutéale. - La durée moyenne de la phase lutéale des cycles œstraux normaux est de 13,3 $\pm 0,7$ jours $(\bar{X} \pm$ s.e.m.). Cependant $2,2 \%$ des cycles œestraux observés ont des phases lutéales dont la durée moyenne est égale à $24,9 \pm 3,3$ jours $(\bar{X} \pm$ s.e.m.) (tabl. 1). Cet allongement de la phase lutéale est constaté chez $14 \%$ des animaux

\section{TABLEAU 1}

Durées et périodes d'apparition des phases lutéales prolongées chez 3 brebis peulhs bicolores.

\begin{tabular}{ccl}
$\begin{array}{c}\text { Numéro } \\
\text { brebis }\end{array}$ & $\begin{array}{c}\text { Durée des phases lutéales } \\
\text { (jours) }\end{array}$ & $\begin{array}{c}\text { Période de l'année } \\
\text { concernée }\end{array}$ \\
\hline 366 & 47 & avril/mai \\
366 & 19 & février \\
366 & 18 & juillet/août \\
366 & 24 & septembre \\
366 & 26 & octobre \\
366 & 24 & mars \\
110 & 21 & avril \\
115 & 20 & octobre \\
\hline
\end{tabular}

TABLEAU 2

Durées et périodes d'apparition de l'anoestrus chez 16 brebis peulhs bicolores.

\begin{tabular}{|c|c|c|}
\hline $\begin{array}{l}\text { Numéro } \\
\text { brebis }\end{array}$ & $\begin{array}{c}\text { Durée de l'anœstrus } \\
\text { (jours) }\end{array}$ & $\begin{array}{l}\text { Période de l'année } \\
\text { concernée }\end{array}$ \\
\hline 361 & 45 & octobre/novembre \\
\hline 362 & 46 & décembre/février \\
\hline 362 & 72 & février/avril \\
\hline 364 & 133 & janvier/mai \\
\hline 364 & 95 & juin/septembre \\
\hline 8004 & 152 & janvier/mai \\
\hline 366 & 57 & décembre/février \\
\hline 366 & 51 & février/avril \\
\hline 102 & 125 & janvier/avril \\
\hline 104 & 167 & décembre/mai \\
\hline 104 & 33 & juin/juillet \\
\hline 110 & 36 & janvier/février \\
\hline 111 & 156 & décembre/mai \\
\hline 111 & 37 & mai/juin \\
\hline 112 & 151 & janvier/mai \\
\hline 112 & 53 & mai/juillet \\
\hline 112 & 51 & juillet/septembre \\
\hline 115 & 129 & juillet/mai \\
\hline 119 & 39 & janvier/février \\
\hline 119 & 27 & mars/avril \\
\hline 127 & 39 & janvier/février \\
\hline 201 & 144 & décembre/mai \\
\hline 209 & 65 & janvier/mars \\
\hline 217 & 62 & février/mai \\
\hline \multicolumn{3}{|c|}{$\bar{x} \pm$ s.e.m. $=81,9 \pm 9,8$ jours } \\
\hline
\end{tabular}


et apparaît au cours des périodes février/mai et août/novembre. La brebis $n^{\circ} 366$ manifeste plus fréquemment cette anomalie.

3) Variations et importance de l'allongement de l'intervalle entre deux phases lutéales consécutives. - Les cycles ostraux normaux ont une durée moyenne de $16,79 \pm 0,06$ jours $(\bar{X} \pm$ s.e.m. $)$; leurs phases lutéales sont séparées par des intervalles qui ont une durée moyenne de 4,3 $\pm 0,2$ jours.

Du fait des arrêts de l'activité cyclique du comportement d'œstrus au cours de la période de janvier à avril, les cycles œstraux ont une durée moyenne de $81,9 \pm 9,8$ jours ( $\bar{X} \pm$ s.e.m.) (tabl. 2). Ce phénomène a été observé chez $71 \%$ des animaux et on peut enregistrer jusqu'à 3 cycles de ce type chez une brebis.

$\mathrm{Au}$ cours de ces cycles allongés, des ovulations silencieuses ont lieu fréquemment, et l'intervalle entre la fin d'une phase lutéale et le début de la suivante est au moins supérieur à 5 jours. Dans $79 \%$ des cas, cette durée est comprise entre 5 et 29 jours (tabl. 3). Cet allongement de l'intervalle entre deux phases

TABLEAU 3

Fréquence d'observation de la durée qui sépare deux phases lutéales.

\begin{tabular}{cccccccc}
\hline $\begin{array}{c}\text { Classes d'intervalle }\left(^{*}\right) \\
\text { (jours) }\end{array}$ & $0-4$ & $5-9$ & $10-14$ & $15-19$ & $20-24$ & $25-29$ & $\geq 30$ \\
\hline Fréquence des observations (\%) $\ldots . .$. & 88,8 & 2,3 & 1,4 & 3,2 & 1,7 & 0,3 & 2,3 \\
\hline
\end{tabular}

$\left(^{*}\right)$ Les intervalles ont été regroupés en classes dont la largeur est de 4 jours.

TABLEAU 4

Classement de la durée (en jours) de l'intervalle entre deux phases lutéales en fonction du rang $d^{\prime}$ apparition et de l'animal ${ }^{*}$ ).

\begin{tabular}{|c|c|c|c|c|c|}
\hline \multicolumn{6}{|c|}{ Rang } \\
\hline $\begin{array}{c}\text { Numéro } \\
\text { Brebis }\end{array}$ & 1 & 2 & 3 & 4 & 5 \\
\hline 362 & 16 & 16 & 9 & & \\
\hline 364 & 17 & 44 & 15 & 16 & 24 \\
\hline 8004 & 7 & 71 & 18 & & \\
\hline 111 & 93 & 19 & 7 & & \\
\hline 112 & 10 & 68 & 18 & 22 & 24 \\
\hline 115 & 45 & 39 & 8 & & \\
\hline 201 & 12 & 22 & 8 & 21 & 19 \\
\hline
\end{tabular}

$\left.{ }^{*}\right)$ Les animaux considérés sont ceux qui présentent au moins 3 allongements d'un intervalle de temps compris entre deux phases lutéales. 
lutéales consécutives peut apparaître au maximum 5 fois chez une brebis au cours de la période de janvier à avril; il a durée relativement constante $(P>0,05)$ au cours des apparitions successives (tabl. 4). Les intervalles allongés représentent $11 \%$ des intervalles qui séparent deux phases lutéales consécutives.

4) Importance des ovulations silencieuses. - Les ovulations silencieuses représentent $12 \%$ des ovulations totales enregistrées; $75 \%$ ont lieu entre les mois de janvier et mai, soit en début et en fin de la période d'anœstrus. Des ovulations silencieuses ont lieu tout au long de l'année à l'exception des mois de septembre et octobre où l'œstrus et l'ovulation sont normalement associés (fig. 1).

\section{Expérience II.}

109 endoscopies ont été réalisées et le nombre moyen d'ovulations est de $1,3 \pm 0,04(\bar{X} \pm$ s.e.m.). Il y a alternance de fonctionnement des deux ovaires (le nombre total de séries étudiées par brebis varie de 5 à 19), et le nombre total de corps jaunes enregistrés sur les ovaires droit et gauche est pratiquement identique (72: 71). II n'y a pas de variation significative au seuil $5 \%$ du taux d'ovulations moyen entre la période d'anœstrus (janvier à avril) et la saison sexuelle (mai à décembre).

\section{Discussion.}

Le taux d'ovulations moyen observé chez la brebis peulh bicolore est très voisin de celui de certaines races de brebis marocaines (Beni Ahsen $=1,25$; Timhadit $=1,0$; Lahlou-Kassi et Marie, 1981), australiennes (Merinos $=1,1$; Oldham, 1980) et européennes (lle-de-France $=1,4$; Oldham, 1980: Blackface $=1,3$; Wheeler et Land, 1977), mais plus faible que celui des brebis Romanov $(2,6$; Land et al., 1973) ou Finnoises (4,03 ; Hanrahan et Quirke, 1975). Ce taux d'ovulations ne peut pas être considéré de façon absolue comme caractéristique de la race peulh, compte tenu du faible effectif d'animaux étudié et des conditions d'élevage plus favorables que celles généralement rencontrées au Niger.

II n'y a pas de variation saisonnière significative du taux d'ovulations contrairement aux résultats obtenus chez la brebis finnoise par Wheeler et Land (1977) et la brebis Romanov par Land et al. (1973), ces deux races ayant des taux d'ovulations élevés. Chez la brebis Solognote et Aragonaise à taux d'ovulations $(1$ à 1,2) comparable à celui de la brebis peulh, Land et al. (1973) et Valls et al. (1977) n'observent pas de variation saisonnière de ce paramètre. II est probable que le faible taux d'ovulations observé chez ces races masque la variation saisonnière de I'activité ovarienne puisque les hybrides Aragonaise $\times$ Romanov et Aragonaise $\times$ Finnoise manifestent cette variation. II est cependant possible que la relative constance de la photopériode au cours de l'année soit la cause de ce phénomène.

L'existence d'une activité cyclique de l'ovaire interrompue par un allongement de la phase lutéale et/ou de l'intervalle entre deux phases lutéales consécutives est confirmée chez la brebis bicolore du Niger. Le comportement cyclique d'œstrus présente des variations saisonnières, mais au niveau d'un troupeau, il y a 
au moins $48 \%$ des brebis qui présentent des œstrus et des ovulations toute l'année. Il existe donc une période d'activité sexuelle minimale de janvier à avril, et une période d'activité sexuelle maximale de mai à décembre. Cependant au cours de la pleine' saison sexuelle, les pourcentages d'œestrus et d'ovulations enregistrés $n^{\prime}$ atteignent jamais $100 \%$ hormis pendant les mois de septembre et octobre. Ces 2 derniers mois sont ceux qui présentent le moins d'anomalies de l'activité orarienne.

L'anœstrus observé chez la brebis peulh placée dans de bonnes conditions d'alimentation apparaît à une époque où la photopériode et la température sont croissantes (Yenikoye et al., 1982). Ceci est conforme à ce qui est habituellement observé dans l'hémisphère Nord chez les autres races ovines. Néanmoins, dans les conditions d'élevage traditionnel au Niger où les disponibilités alimentaires sont réduites, la proportion des brebis en œstrus toute l'année peut être moins importante (Guerra et al., 1972) et la durée de l'ancestrus plus longue (Haresign, 1981). II en est probablement de même de l'allongement de la phase lutéale dont l'importance peut dépendre des conditions d'élevage.

L'allongement de la phase lutéale observé chez la brebis peulh correspond à un diœstrus prolongé. II est dû à l'existence de corps jaunes persistants (Yenikoye et al., 1982) attribués à une insuffisance de synthèse et de libération de $\mathrm{PGF}_{2 \alpha}$ par l'utérus (Stabenfeldt et al., 1980), les œstrogènes et l'ocytocine qui influencent cette libération étant eux-mêmes insuffisants (Sheldrick et al., 1980). II est possible aussi que ce diœstrus prolongé soit dû à une déficience en gonadotropines (Restall et al., 1978).

L'anœstrus observé chez la brebis peulh est fréquemment interrompu par des ovulations silencieuses et irrégulières. Ainsi l'intervalle qui sépare deux phases lutéales au cours de cette période correspond dans $79 \%$ des cas à une phase anovulatoire qui dure 5 à 29 jours après la fin de la lutéolyse. Ce type d'ancestrus peut-il être considéré comme une caractéristique de la brebis peulh bicolore puisqu'il apparaît chez $71 \%$ des animaux ? L'absence du comportement d'œstrus qui marque le début de la période anovulatoire peut être due à une insuffisance de sécrétion des œstrogènes par l'ovaire au début de la lutéolyse (Goodman, 1978). L'allongement de l'intervalle qui sépare deux phases lutéales peut être lié à un ralentissement de la maturation folliculaire et/ou à un retard de la décharge préovulatoire de LH (Yenikoye et al., 1982).

La présence d'anœestrus fréquemment interrompu par des ovulations silencieuses et irrégulières et le pourcentage d'animaux cycliques qui reste important toute l'année (au moins $48 \%$ ) suggèrent que l'anœstrus qui apparaît chez la brebis peulh est un phénomène physiologique différent de celui qui est observé chez certaines races de brebis où l'ancestrus est marqué par une inactivité totale de l'ovaire (Thimonier et Mauléon, 1969 ; Land et al., 1973. ; Wheeler et Land, 1977).

L'étude portant sur l'effet de l'introduction de béliers sur la cyclicité des brebis en période peu favorable permettra de préciser s'il s'agit d'une simple déficience de la sécrétion pulsatile de LH qui peut être suppléée par l'effet mâle (Martin et al., 1980) ou d'une modification plus profonde de la folliculogenèse. 
Remerciements. - L'auteur remercie MM. Mariana J. C. et Cognié Y. pour leurs suggestions dans la rédaction de ce texte, Madame Fagu et $M$. André D. pour leur assistance technique.

Ce travail a bénéficié en partie de l'aide financière de la Fondation Internationale pour la Science (Suède), et du Ministère Français de la Coopération.

\section{Références}

CONOVER W. J., 1971. Practical nonparametric statistics. John Wiley and Sons Inc, USA, 130-139; $140-150 ; 349-356$.

GOODMAN R. L., 1978. Role of ovarian steroids in the initiation and synchronization of behavioral estrus and the LH surge in the ewe. Biol. Reprod., 18, suppl. 1, 46 A (abstr.).

GUERRA J. C., THWAITES C. J., EDEY T. N., 1972. The effects of components of body weight on reproductive efficiency in merino ewe. J. agri. Sci. Camb, 78, 245-249.

GUNN R. G., DONEY J. M., 1979. Ewe management for control of reproductive performance. ADAS Quart. Rev., 35, 231-245.

HAFEZ E. S. E., 1952. Studies on the breeding season and reproduction of the ewe. J. agri. Sci., 42, 189-265.

HANRAHAN J. P., QUIRKE J. F., 1975. Repeatibility of the duration of oestrus and breed differences in the relationship between duration of oestrus and ovulation rate of sheep. J. Reprod. Fert., 45, 29-36.

HARESIGN W., 1981. The influence of nutrition on reproduction in the ewe. 1. Effects on ovulation rate follicle development and LH release. Anim. Prod., 32, 197-202.

LAHLOU-KASSI A., MARIE M., 1981. A note on ovulation rate and embryonic survival in D'Man ewes. Anim. Prod., 32, 227-229.

LAND R. B., PELLETIER J., THIMONIER J., MAULÉON P., 1973. A quantitative study of genetic differences in the incidence of oestrus, ovulation and plasma LH concentration in the sheep. J. Endocr., 58, 305-317.

MARTIN G. B., OLDHAM C. M., LINDSAY D. R. 1980. Increased plasma LH levels seasonally anovular Merino ewes following the introduction of rams. Anim. Reprod. Sci., 3, 125-132.

MAULĖON P., 1980. Variations circannuelles de la fertilité chez les mammifères domestiques, 15-32. In Rythmes et reproduction. Masson, Paris.

OLDHAM C. M., 1980. A study of sexual and ovarian activity in Merino sheep. Ph. D. Thesis, Univ. Western Australia.

RESTALL B. J., KEARINGS R. D., HERDEGEN J., CARBERRY P., 1978. The induction of reproductive activity in lactating ewes. Aust. $J$. agric. Res., 29, 181-187.

STABENFELDT G. H., NEELY D. P., HUGUeS J. P., KINDAHL H., 1980. Modification of uterine $\mathrm{PGF}_{2 \alpha}$ in domestic animals through pathologic or pharmacologic processes. 9th int. Congr. anim. Reprod. A. l., vol. II, 27-34, Madrid.

SHELDRICK E. L., MITCHELL M. D., FLINT A. P. F., 1980. Delayed luteal regression in ewes immunized against oxytocin. J. Reprod. Fert., 59, 37-42.

THIMONIER J., MAULÉON P., 1969. Variations saisonnières du comportement d'œstrus et des activités ovariennes et hypophysaires chez les ovins. Ann. Biol. anim. Bioch. Biophys., 9, 233-250.

VALLS O. M., COGNIÉ Y., VALDEMORO M. D., HALLAUER J., 1977. El cruce Romanov y Sinès con la raza Rasa Aragonesa. Resultados preliminares sobre el engorde y la reproduccion. Inf. tec. econ. Agrar., 26, 25-30.

WHEELER A. G., LAND R. B., 1977. Seasonal variation in oestrus and ovarian activity of Finnish. Landrace, Tasmanian Merino and Scottish blackface ewes. Anim. Prod., 24, 363-376.

YENIKOYE A., ANDRÉ D., RAVAULT J. P., MARIANA J. C., 1981. Etude de quelques caractéristiques de reproduction chez la brebis peulh du Niger. Reprod. Nutr. Dévelop., 21, $937-$ 951.

YENIKOYE A., PELLETIER J., ANDRE D., MARIANA J. C., 1982. Anomalies in ovarian function of peulh ewes. Theriogenelogy, 17, 355-364. 